\title{
Design and Implementation of Aerial Vehicle Remote Sensing and Surveillance System, Dehazing Technique Using Modified Dark Channel Prior
}

\author{
Hasn Mahmood Khudair ${ }^{1}$, Taif Alawsi ${ }^{2, *}$, Anwaar A. Aldergazly ${ }^{1}$, A. H. Majeed ${ }^{1}$ \\ ${ }^{1}$ Al Nahrain University, Department of Laser and Optoelectronics Engineering, Baghdad, 10001, Iraq \\ ${ }^{2}$ Sapienza University of Rome, Department of Information Engineering, Electronics and Telecommunication, Rome, 00100, Italy
}

\begin{tabular}{l} 
A R T I C L E I N F O \\
\hline Article history: \\
Received: 13 June, 2020 \\
Accepted: 18 September, 2020 \\
Online: 20 October, 2020 \\
\hline Keywords: \\
Unmanned Aerial Vehicle (UAV) \\
Remote Sensing \\
Dehazing \\
Dark Channel Prior (DCP)
\end{tabular}

\section{Introduction}

The presence of haze reduces the perception of the Aerial imagery observed by the captured image in the UAV. Such deterioration affects computer vision systems' efficiency in aerial vehicle applications, including video monitoring, automatic vehicle navigating, and remote sensing. Light dispersion is caused by suspended objects, just like droplets of water or smog that can reduce the visibility in hazy environments. The degree of visibility losses depends upon the intensity and range among view objects and the observational location of the suspended particles in that environment. During the past years, studies have concentrated on creating effective computational methods to eliminate the effects of haze in aerial photogrammetry of the exterior surroundings. For example, the processing of many views captured during various hazy circumstances is an algorithm on which the research is based [1]. The algorithm we modified and implemented in this research is based on the DCP algorithm which is commonly utilized in the defogging algorithm. The processing of the DCP algorithm based on the scattering process resulting from fog and weather circumstances, photographs from low altitudes can be decayed. Such unfavorable environmental conditions reduce the color and

${ }^{*}$ Corresponding Author: Taif Alawsi, Iraq, Baghdad, +9647714202625 \&

taif.alawsi@uniroma1.it

www.astesj.com

https://dx.doi.org/10.25046/aj0505135 contrast of the views and thus its radiometric performance. The article provides a way of image restoration collected from aerial vehicle (AV) sites. Across the course of the study, an algorithm for low-altitude imaging has been developed and environmental adjustment algorithms based on a model for atmospheric dispersion were being shown. Performance testing of the system of dehazing suggested had been performed by only using subjective metrics like the SSIM, PSNR, RMS, normalized crosscorrelation, Universal Image Quality Index, the root mean square error (RMSE). Investigations showed that utilizing the dehazing algorithm, could minimize the adverse effects of haze and enhance the quality of aerial imagery dependent on the PSNR scale [2-5].

\section{Literature Review}

The dark channel prior (DCP) algorithm is considered to be widely used in fog reduction and image enhancement, which was suggested primarily [1]. Such an algorithm separates the scene across hazy and foggy zones. In [6], the author utilized bilateral and denoise processing to obtain that graph of the transmission [7]. In [8], the author was using the edge smoothing filter of the DCP and conducted multiscale acoustic manipulation. In [9], the author suggested a way of utilizing contrast hazy dependence, i.e. fog-free scenes are better over hazy photographs. Due to weather effects, 
lighting, shadows, and occlusion, roads in imagery show its complex characteristics, which make road mining difficult. In [10], the author proposed an automatic method of aerial extraction for a vague Retinex based aerial images and an improved Canny Operator with the shape criteria. The monitoring and line detection method has been researched for invariant illumination images. In [11], the author studied the bioinspired classificatory for remote sensing imaging surveillance mining, which takes into account the dynamic conditions of weather such as hazy and foggy weather.

\section{Theoretical Background}

\subsection{Hazy Environment}

A captured scene in the existence of fog as shown in Figure 1 which can be seen in a uniform medium using an algorithm to atmospheric optics as follows:

$$
I(x)=J(x) t(x)+A(1-t(x))
$$

The formula of transmission function in the medium can be described as follow:

$$
t(x)=\exp (-B d(x))
$$

A definition for all the variables/constants in the equations above can be seen in Table 1. Overall, the problem is the restoration of the reflectance function $\mathrm{J}(\mathrm{x}), \mathrm{t}(\mathrm{x})$, and also $\mathrm{B}$ for the foggy view $\mathrm{I}(\mathrm{x})$. This must be mentioned whether model dehazing is an unrecognized concern since the only single hazy image can approximate the $\mathrm{J}(\mathrm{x})$ functions and also the variables $\mathrm{b}$ and $\mathrm{A}$.
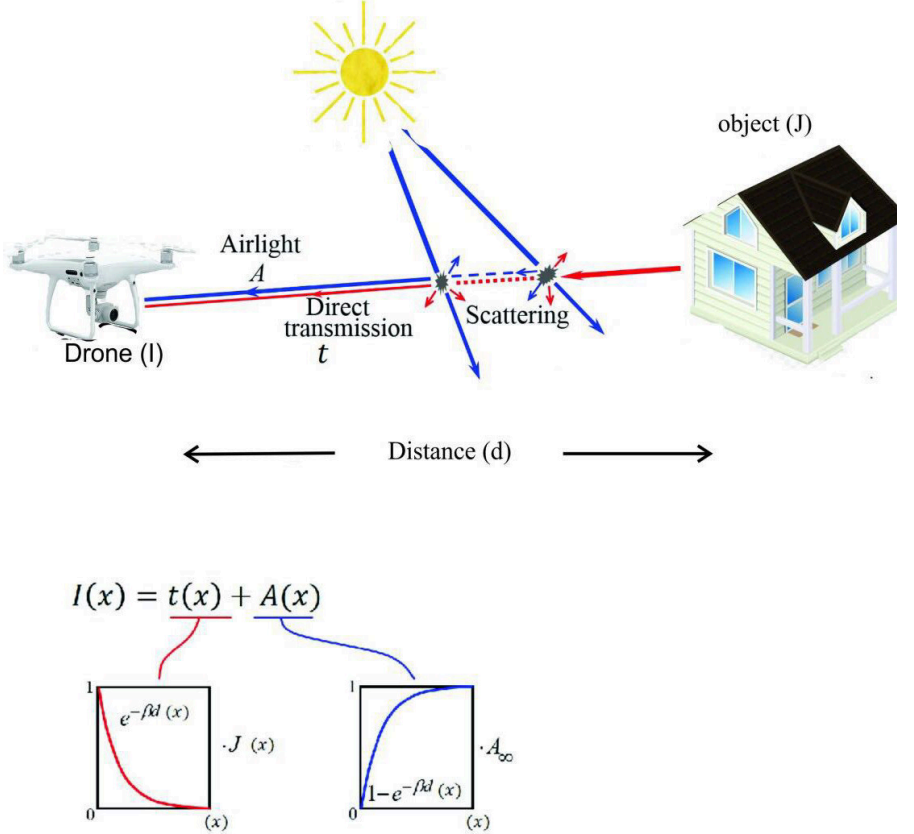

Figure 1: The main scheme of the hazy environment can be affected by the surveillance system

\subsection{Dark Channel Prior (DCP) Algorithm}

The DCP is shown in three RGB color filters via calculating the minimum spatial illumination values for the bright object, accordingly [9]:

$$
D C(x)=\min _{c \in\{r, g, b\}}\left\{\min _{x \in \Omega_{s q}}\left\{\frac{I^{c}(x)}{\hat{A}^{c}}\right\}\right\}
$$

The DCP algorithm considered the most widely used method of dehazing algorithms which can be illustrated as following [12]

1) A simplistic, rational, clear, and true inference is derived from an outdoor natural picture of statistical characteristics.

2) No modification to dehazing is made before the collection of the initial meaning variables are calculated.

Table 1: Mapping the definition of each symbol in the equations

\begin{tabular}{|c|c|}
\hline Symbol & Quantity \\
\hline $\mathrm{I}(\mathrm{x})$ & Single hazy image \\
\hline$A$ & Scalar constant (airlight) \\
\hline$t(x)$ & Medium transmission function \\
\hline$J(x)$ & $\begin{array}{c}\text { Reflectance function of the scene (haze-free } \\
\text { image) }\end{array}$ \\
\hline$d(x)$ & Scene depth from the camera viewpoint \\
\hline$\beta$ & $\begin{array}{c}\text { Attenuation coefficient determined by the } \\
\text { weather condition }\end{array}$ \\
\hline$\Omega$ & $\begin{array}{c}\text { A subset of pixel coordinates defining a square } \\
\text { structure around the central coordinates x. }\end{array}$ \\
\hline MSE & Mean Square Error \\
\hline RMSE & Root Mean Square Error \\
\hline PSNR & Peak Signal to Noise Ratio \\
\hline
\end{tabular}

\subsection{Suggested Dark Channel Prior Algorithm}

A modified DCP algorithm was suggested in this article for the surveillance system in foggy/hazy/dusty weather conditions, which is illustrated in Figure 2.A. The input image has the desired criteria to be enhanced using the DCP algorithms, these criteria were a foggy indoor image, foggy outdoor image, and dusty outdoor image. After capturing the images in the UAV with the desired conditions, these images were processed by MATLAB ${ }^{\circledR}$ to calculate the image quality parameters/metrics. Then applying the DCP algorithms to dehaze/defog the images and then again calculate the parameter/metrics for the dehazed image. The final step will be the optimization of the parameters/metrics and then getting the final output of the dehazed images, as shown in Figure 2.B. The procedure described above was repeated for three different algorithms based on the DCP. The snapshots of the MATLAB ${ }^{\circledR}$ programming for image dehazing is shown in Figure 3.

Mainly based on five steps described in the experimental implementation section in this article. We modified the DCP algorithm by calculating the attenuation coefficient of different images under different hazy conditions then varying the attenuation coefficient $(\alpha)$ until the images were dehazed and enhanced the resolutions by varying and modifying the DCP algorithm to obtain modified DCP used in different hazy conditions in different heights. 


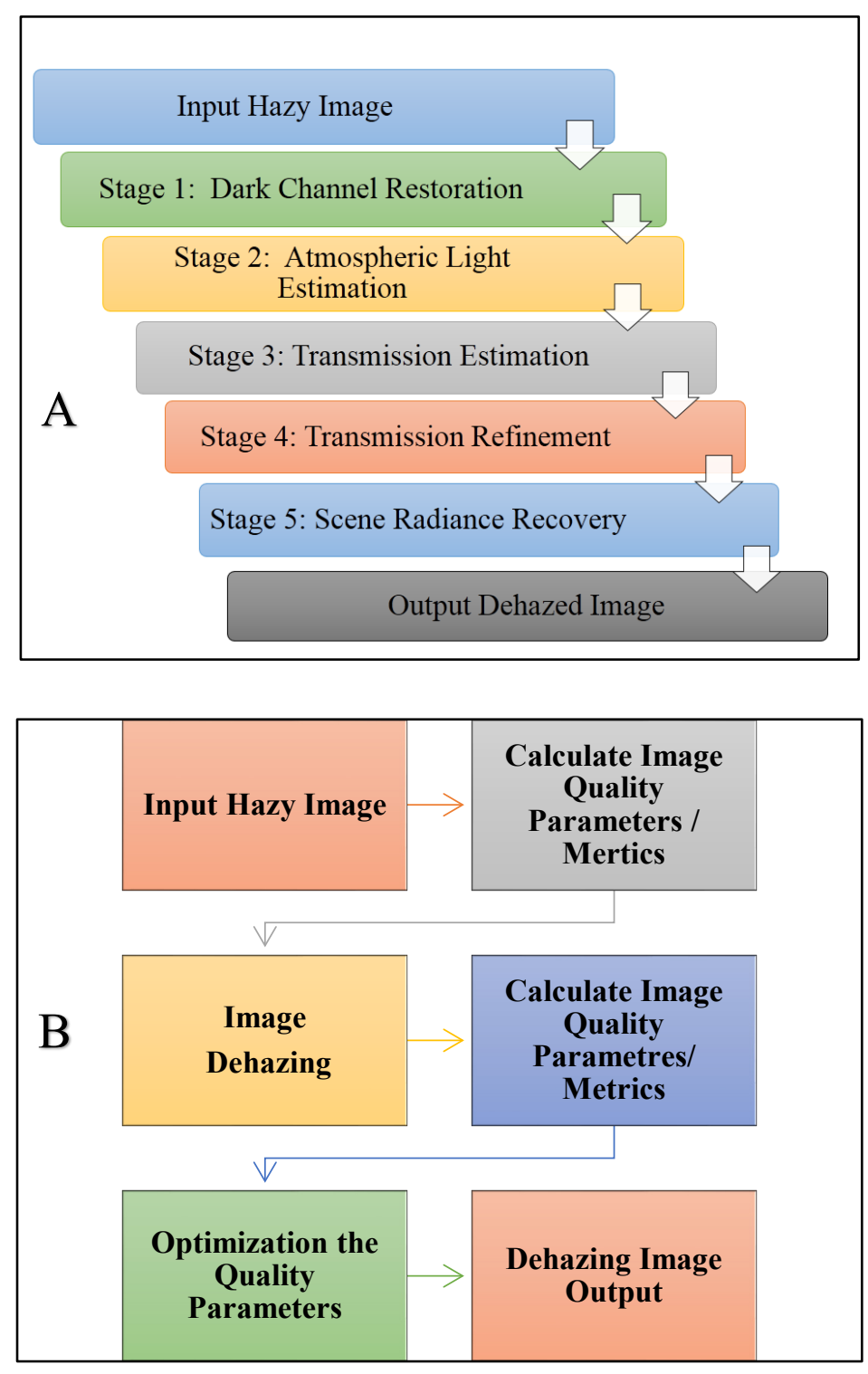

Figure 2: Schematic illustration for the DCP algorithm (a) Steps for the DCP algorithm (b) Dehazing workflow for our suggested DCP algorithm
Input Image

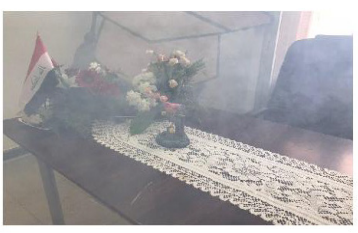

Initial Transmission

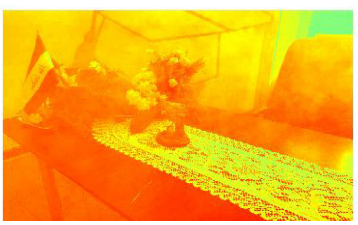

Recovered Image

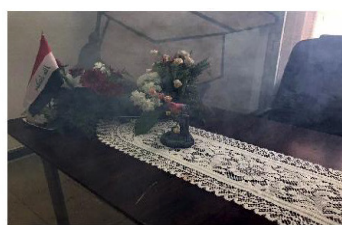

Estimated Transmission

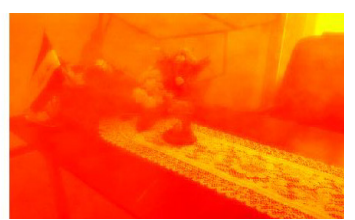

Figure 3: The DCP approach on an experimental image captured in our lab
Utilizing the atmospheric scattering model, the system reconstructs an image from a hazy scene. A general dehazing parameter is used by the process to modify the degree of haze removal so that the image does not look over defogging. An effective methodology to dehaze an image using modified DCP as shown in Figure 2.

\section{Dark channel restoration}

It is presumed that one channel (red, green, or blue) is usually very dark, except for the sky, with a given pixel in a color image of a natural scene. Which allows for accurate measurement of the dark channel. The dark channel $\mathrm{J}^{\mathrm{dark}}(\mathrm{x})$ is for a hazy image $\mathrm{J}(\mathrm{x})$ in the following equation:

$$
J^{\text {dark }}(x)=\min _{c \in\{r, g, b\}}\left\{\min _{y \in \Omega(x)}\left\{J^{c}(y)\right\}\right\}
$$

where: $\mathrm{J}^{\mathrm{c}}$ is a color channel of $\mathrm{J}$ and $\mathrm{y}$ is a local patch centered at $\mathrm{x}$.

\section{Atmospheric light estimation}

When the atmosphere is gloomy and the sunlight (S) is overlooked and even the ambient light $\left(A_{\infty}\right)$ is considered the brightest pixel in an eyelid is considered to be the strongest eyelid. Thus, the brightest pixel can be brighter than the atmospheric light by utilizing the following equation:

$$
\begin{gathered}
J(x)=R(x)\left(S+A_{\infty}\right) \\
I(x)=R(x) S t(x)+R(x) A_{\infty} t(x)+(1-t(x)) A_{\infty} \geq A_{\infty}
\end{gathered}
$$

Where: $A_{\infty}$ is the brightest pixels in an eyelid image intensity without haze is ranging between $0 \leqslant \mathrm{R} \leqslant 1$.

\section{Transmission estimation}

Transmission is estimated as follows after estimating the atmospheric light

$$
t(x)=1-\omega \min _{y \in \Omega(x)}\left\{\min _{c}\left\{\frac{I^{c}(y)}{A_{\infty}^{c}}\right\}\right\}
$$

Where: $\omega$ is the haze kept in the photo to prevent unnatural scenes $(\omega=0.95)$.

\section{Transmission Refinement}

Soft matting Laplacian [11] is applied to smooth objects along the edges to refine the transmission map. It dramatically increases the computing time.

\section{Scene Radiance Recovery}

Which may deduce that we apply to the Equation:

$$
J(x)=\left(\frac{I(x)-A_{\infty}}{\max \left(t(x), t_{0}\right)}\right)+A_{\infty}
$$

We set $t_{0}=0.1$ so that the dimly enhanced image will be avoided.

The optimization of our modified DCP technique was obtained by testing the quality of captured images to get the attenuation 
coefficient $(\alpha)$ which referred to the haze in the scene. Then the image is processed and by varying the attenuation coefficient $(\alpha)$ values we aim to get better results in dehazing. The values of $\alpha$ that have been taken in our algorithm are three values $(\alpha=0.5,0.7$, 0.9 ) and we compared our results with previous DCP algorithms.

\section{Experimental Work}

\subsection{Images Capturing Setup}

The experimental setup used for capturing the desired foggy/hazy/dusty scene in this research employed the following tools:

UAV: The UAV used was the Phantom 4 Pro model, produced by Dji co. ${ }^{\circledR}$ (China), with high specifications and camera quality. The UAV is equipped with a 20 Mpix camera as well as an AV navigation system in addition to a variety of other features relevant to the research at hand, such as snapshots, good battery life, GPS, and easy-to-control joysticks, as shown in Fig 4.A. The purpose of using the UAV in this research is primarily to scan the designated terrain, capture photographs in foggy, hazy, and dusty conditions to be used for image analysis and processing by the dark channel prior algorithm.

Fogger: Is utilized for generating foggy-like environmental circumstances. By adding the fogging liquid that consists of a chemical material which turns into a white water vapor due to heating initiated by the fogger. The reason for using the fogger is to generate the fog required to obtain a foggy condition essential for the reconnaissance, which can be very useful in indoor conditions, as shown in Figure 4.B.

The room (Lab): We used two different rooms with a $5 \times 6 \mathrm{~m}^{2}$ area and $5 \times 4 \mathrm{~m}^{2}$, where the heights are equal for both rooms $(3 \mathrm{~m})$ suitable for photography and reconnaissance as shown in Figure 4.A, and Figure 5.

The software used in our research are as follows:

- MATLAB $^{\circledR}$ software was utilized for aerial photogrammetry processing with DCP algorithm implementation.

- UAV Deploy ${ }^{\circledR}$ and DJI Go ${ }^{\circledR}$ applications are utilized as a UAV pilot to control and capture the scene, as shown in Figure 4.C.
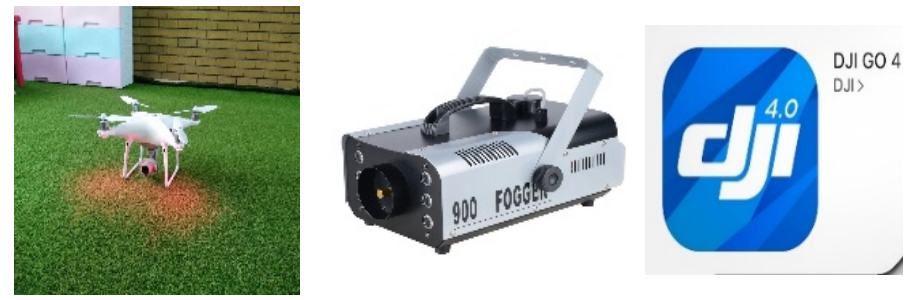

Figure 4: The experimental setup used in this work (a) UAV (b) Fogger (c) DGI $\mathrm{GO}^{\circledR}$ App

Our suggested algorithm is obtained by using the UAV (Phantom 4 Pro $V_{2}$ ) that has the modern specifications to uptake the surveillance. This is done by gathering the information by capturing images in different foggy/hazy/dusty conditions. The images were then processed in MATLAB ${ }^{\circledR} 2019$ with different DCP algorithms and were processed. We have selected the value of attenuation coefficients that give the clearest scenes for images haze removal and then enhance the dehazing image.

\subsection{Experimental Implementation}

The equipment used in this research was installed as was described in the previous section. After that the fogger was switched on after the addition of the fogging substance, the UAV was launched at the given designated route for reconnaissance and the UAV was programmed to take one photograph per second. We programmed the UAV to take photographs at various heights ( $3 \mathrm{~m}, 25 \mathrm{~m}$, and $10 \mathrm{~m}$ for the foggy indoor, dusty outdoor, foggy outdoor images, respectively) with the presence of fog in the research environment. After conducting the reconnaissance and collecting the photographs, we have transferred the photos from the UAV's memory card to the PC for photo rendering to be enhanced and conduct our calculation (Image quality metrics, normalized cross-correlation, and (PSNR/RMSE)). We processed the images using MATLAB ${ }^{\circledR}$ software by inserting the suggested algorithm that we have developed to enhance the image and remove the fog. The images were enhanced and the fog was removed by inserting two previous algorithms to compare the result as well as developing future researches to address some of the weak points of such studies. Two previous articles were chosen to compare their results after applying the captured images in the previously chosen algorithms. The calculation for Image Quality parameters/metrics; normalized cross-correlation, (PSNR), Average Difference, Structure Content, Max Difference, Normalized Absolute Error then the results of our calculations were compared and contrasted with QFP of our algorithm and the chosen scene as is shown in Figure 5.

\section{Results}

The output dehazed images obtained from the suggested algorithm on the selected images are shown below with a comparison of two other DCP algorithms [13]. The images were selected in various weather conditions as shown in Figure 6 under DCP, we also calculated the image quality parameters/metrics and compared them with our modified algorithm. The algorithms used in the comparison are $\mathrm{He}$, et al [14] algorithm and Fattal's [15] algorithm. The selected circumstances that we have been testing our modified DCP are foggy indoor image, as shown in Figure 6.A, dusty outdoor image, as presented in Figure 6.B, and dusty outdoor image, as depicted in Figure 6.C, respectively. The altitude of the images A, B, and C are 3, 25, and $10 \mathrm{~m}$, respectively. Images in Figure 6 were processed by three different DCP algorithms, the input images have different conditions of haze/fog and therefore different results are shown for each image. Our method gives more enhancement and clearer output in comparison with the other DCP algorithms.

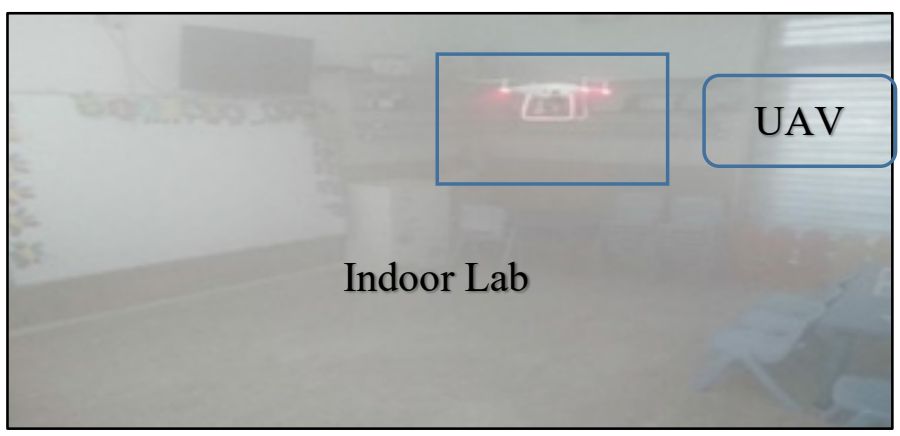

Figure 5: Experimental implementation setup of our study for the hazy environment using UAV in indoor conditions 


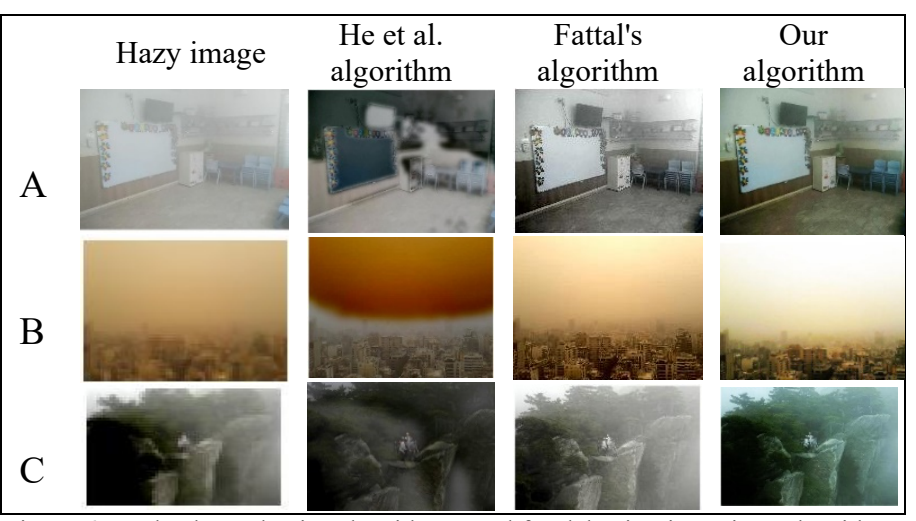

Figure 6: Dark Channel Prior algorithms used for dehazing in various algorithms in three different hazy circumstances (images from the experimental setup) (a) Indoor foggy image (b) Outdoor dusty image (c) Outdoor foggy image

Table 2 below provides the comparative calculations of image quality parameters/metrics and the comparison between these values which are discussed in the discussion section. The image quality parameters/metrics were calculated using MATLAB $^{\circledR}$ software.

Other comparative results for the DCP algorithms were used for images obtained from Google ${ }^{\circledR}$ with foggy and dusty environmental conditions, the altitude of the images $\mathrm{A}, \mathrm{B}$, and $\mathrm{C}$ are $80,125,50 \mathrm{~m}$, respectively, as shown in Figure 7. The image quality metrics/parameters for these sets of images are presented in Table 3.

Table 2: The $\mathrm{Q}$ indices for the images $\mathrm{A}, \mathrm{B}$, and $\mathrm{C}$, respectively

\begin{tabular}{|c|c|c|c|}
\hline $\begin{array}{l}\text { Image Q } \\
\text { indices }\end{array}$ & $\begin{array}{c}\text { He et al } \\
\text { algorithm }\end{array}$ & $\begin{array}{c}\text { Fattal's } \\
\text { algorithm }\end{array}$ & $\begin{array}{c}\text { Our } \\
\text { algorithm }\end{array}$ \\
\hline \multicolumn{4}{|c|}{ Image (A) (Foggy Indoor) (Altitude 3 m) } \\
\hline MSE & $8.43 \mathrm{e}+03$ & $3.07 \mathrm{e}+03$ & $2.69 \mathrm{e}+03$ \\
\hline PSNR & 8.87 & 13.25 & 13.84 \\
\hline $\begin{array}{l}\text { Normalized } \\
\text { Cross- } \\
\text { Correlation }\end{array}$ & 0.58 & 0.82 & 0.86 \\
\hline $\begin{array}{c}\text { Average } \\
\text { Difference }\end{array}$ & 68.53 & 37.15 & 35.85 \\
\hline $\begin{array}{c}\text { Structure } \\
\text { Content }\end{array}$ & 2.28 & 1.36 & 1.27 \\
\hline Max Difference & 236 & 255 & 146 \\
\hline $\begin{array}{c}\text { Normalized } \\
\text { Absolute Error }\end{array}$ & 0.41 & 0.25 & 0.18 \\
\hline \multicolumn{4}{|c|}{ Image (B) (Dusty Outdoor) (Altitude $25 \mathrm{~m}$ ) } \\
\hline MSE & $5.25 \mathrm{e}+03$ & $1.14 \mathrm{e}+03$ & $1.89 \mathrm{e}+03$ \\
\hline PSNR & 10.93 & 17.58 & 15.36 \\
\hline $\begin{array}{l}\text { Normalized } \\
\text { Cross- } \\
\text { Correlation }\end{array}$ & 0.57 & 0.78 & 0.86 \\
\hline $\begin{array}{c}\text { Average } \\
\text { Difference }\end{array}$ & 55.65 & -11.84 & -22.56 \\
\hline $\begin{array}{c}\text { Structure } \\
\text { Content }\end{array}$ & 2.69 & 0.76 & 0.78 \\
\hline Max Difference & 115 & 117 & 64 \\
\hline $\begin{array}{c}\text { Normalized } \\
\text { Absolute Error }\end{array}$ & 0.42 & 0.21 & 0.18 \\
\hline
\end{tabular}

\begin{tabular}{|c|c|c|c|}
\hline MSE & $2.23 \mathrm{e}+03$ & $1.84 \mathrm{e}+03$ & $1.64 \mathrm{e}+03$ \\
\hline PSNR & 14.64 & 15.49 & 15.98 \\
\hline $\begin{array}{c}\text { Normalized } \\
\text { Cross- } \\
\text { Correlation }\end{array}$ & 0.53 & 0.82 & 0.87 \\
\hline $\begin{array}{c}\text { Average } \\
\text { Difference }\end{array}$ & 38.2 & -32.19 & -32.34 \\
\hline $\begin{array}{c}\text { Structure } \\
\text { Content }\end{array}$ & 3.01 & 0.54 & 0.51 \\
\hline Max Difference & 162 & 118 & 111 \\
\hline $\begin{array}{c}\text { Normalized } \\
\text { Absolute Error }\end{array}$ & 0.47 & 0.38 & 0.38 \\
\hline
\end{tabular}

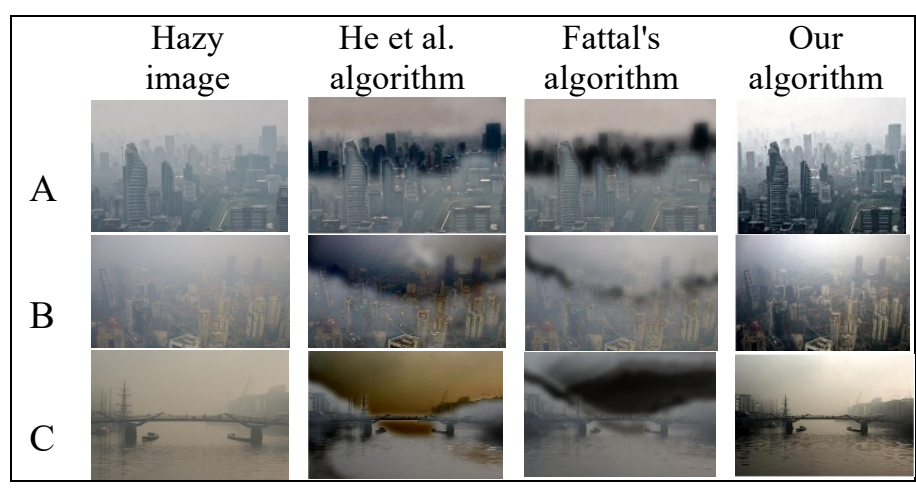

Figure 7: Dark Channel Prior algorithms used for dehazing in various algorithms in three different hazy circumstances with different altitudes (images from Google ${ }^{\circledR}$ ) (a) $80 \mathrm{~m}$ (b) $125 \mathrm{~m}$ (c) $50 \mathrm{~m}$

Figure 8 shows a selective comparison for the metrics of the three processed images of Fig, 6.A, 6.B, and 6.C, respectively, the comparison was done with our method and two previous methods for (a) PSNR (b) Normalized Absolute Error.
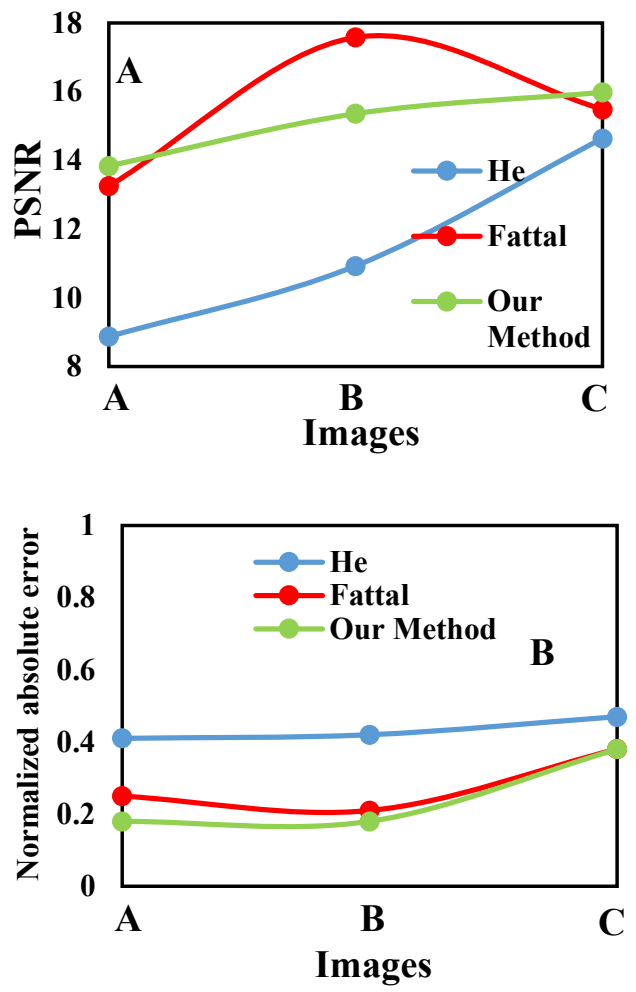

Figure 8: Comparison of the DCP methods (a) PSNR (b) Normalized absolute error 
Table 3: The $\mathrm{Q}$ indices for the images $\mathrm{A}, \mathrm{B}$, and $\mathrm{C}$, respectively

\begin{tabular}{|c|c|c|c|}
\hline Image $Q$ indices & $\begin{array}{c}\text { He et al } \\
\text { algorithm }\end{array}$ & $\begin{array}{c}\text { Fattal's } \\
\text { algorithm }\end{array}$ & $\begin{array}{c}\text { Our } \\
\text { algorithm }\end{array}$ \\
\hline \multicolumn{4}{|c|}{ Image (A) (Altitude 80 m) } \\
\hline MSE & $3.32 \mathrm{e}+03$ & $3.25 \mathrm{e}+03$ & $2.94 \mathrm{e}+03$ \\
\hline PSNR & 12.92 & 13.01 & 13.44 \\
\hline $\begin{array}{c}\text { Normalized } \\
\text { Cross-Correlation }\end{array}$ & 0.72 & 0.75 & 0.87 \\
\hline $\begin{array}{c}\text { Average } \\
\text { Difference }\end{array}$ & 37.5 & 35.95 & 35.1 \\
\hline Structure Content & 1.67 & 1.62 & 0.9 \\
\hline Max Difference & 174 & 180 & 254 \\
\hline $\begin{array}{c}\text { Normalized } \\
\text { Absolute Error }\end{array}$ & 0.28 & 0.28 & 0.26 \\
\hline \multicolumn{4}{|c|}{ Image (B) (Altitude 125 m) } \\
\hline MSE & $4.61 \mathrm{e}+03$ & $5.11 \mathrm{e}+03$ & $2.3 \mathrm{e}+03$ \\
\hline PSNR & 11.5 & 11 & 14.5 \\
\hline $\begin{array}{c}\text { Normalized } \\
\text { Cross-Correlation }\end{array}$ & 0.63 & 0.62 & 0.94 \\
\hline $\begin{array}{c}\text { Average } \\
\text { Difference }\end{array}$ & 54.54 & 50.18 & 13.64 \\
\hline Structure Content & 2.37 & 2.05 & 1.03 \\
\hline Max Difference & 164 & 167 & 255 \\
\hline $\begin{array}{c}\text { Normalized } \\
\text { Absolute Error }\end{array}$ & 0.39 & 0.41 & 0.19 \\
\hline \multicolumn{4}{|c|}{ Image (C) (Altitude 50 m) } \\
\hline MSE & $3.86 \mathrm{e}+03$ & $2.13 \mathrm{e}+03$ & $2.61 \mathrm{e}+03$ \\
\hline PSNR & 12.26 & 12.52 & 13.95 \\
\hline $\begin{array}{c}\text { Normalized } \\
\text { Cross-Correlation }\end{array}$ & 0.62 & 0.72 & 0.84 \\
\hline $\begin{array}{c}\text { Average } \\
\text { Difference }\end{array}$ & 50.18 & 54.18 & 1.83 \\
\hline Structure Content & 2.2 & 2 & 0.9 \\
\hline Max Difference & 148 & 187 & 255 \\
\hline $\begin{array}{c}\text { Normalized } \\
\text { Absolute Error }\end{array}$ & 0.37 & 0.49 & 0.22 \\
\hline
\end{tabular}

\section{Discussion}

From the previous experimental work and their implementation, the output dehazed images obtaining from the DCP algorithm on the selected images as shown in Figure 6 and Figure 7 using the image capturing setup and images in Google ${ }^{\circledR}$ which includes three DCP algorithms. These results illustrate the differences between using the DCP algorithms that have been developed over the years. Our modified DCP algorithm that has been developed gave high image quality in outer photogrammetry that used in monitoring systems in hazy weather such as foggy and dusty environments. In calculations obtained using our algorithm, noticing that the values in indoor hazy aerial photogrammetry (Table 2, Figure 6.A), the MSE, average difference, structure content, max difference, and normalized absolute error values reduced respectively while the PSNR and the normalized cross-correlation values raised to give good quality and eliminate the haze effect on the imagery. In the dusty weather test (Table 2, Figure 6.B), the MSE, average difference, max difference, normalized cross-correlation, and the normalized absolute error values were reduced by using our modified DCP algorithm while PSNR and structure content better in Fattal's algorithm. For outdoor hazy aerial image (Table 2, Figure 6.C), the MSE, average difference, structure content, and max difference values respectively reduced. While there are comparatively increasing in the other parameters. Even if the images were taken apart from the setup as shown in Figure 7, the suggested algorithm is enhancing the image and removing the fog and dust in the surrounding environment and producing a clear view of the scene (Table 3, Figure 7.A, Figure 7.B, Figure 7.C).

The restriction and other related algorithms based upon statistics of the proposed method in which image values with particular images, e.g., obtained in very different examples cannot be used (harsh weather conditions). If only part of the object in the picture is obscured by heavy fog, using the algorithm would distort the image's color reproduction and degrade as a result affects the spectral image quality which utilized radiometric correction with low impact. The atmosphere is important for the accuracy of subsequent remote sensing analyses in low altitudes; therefore, calibration panels with a proven reflection coefficient should be used to check device calibration in the processed image and estimate radiometric error. Also, geophysical limitations are not included in the proposed method, for example, variables like humidity.

\section{Conclusions}

This paper demonstrates the results of research into low altitude dehazing picture methods. The DCP algorithm has been used to eliminate the haze induced by the negative effect of the low atmosphere on UAV images. The variables of our algorithm were empirically determined. We have a strategy dedicated to low altitude photos, in particular for the most commonly used altitudes (in the range below 30 meters) for remote sensing with the UAV. The efficiency of the system proposed was assessed and many qualitative metrics that were used to establish the effectiveness of our suggested algorithm. In comparison with other common methods used for dehazing images, the results of our experiments showed the effectiveness of our process. Moreover, the image data collection in various heights and atmospheric conditions is obtained by careful assessment of weather conditions using the UAV. We have shown that our approach is of significant value in terms of improving the image quality in different weather conditions.

\section{Conflict of Interest}

The authors declare no conflict of interest

\section{Acknowledgment}

We would like to acknowledge the department of laser and optoelectronics engineering at Al Nahrain University.

\section{References}

[1] K. He, J. Sun, X. Tang, "Single image haze removal using dark channel prior," IEEE Transactions on Pattern Analysis and Machine Intelligence, 33(12), 2341-2353, 2011, doi:10.1109/TPAMI.2010.168.

[2] A. Mazur, M. Kacprzak, K. Kubiak, J. Kotlarz, K. Skocki, "The influence of atmospheric light scattering on reflectance measurements during photogrammetric survey flights at low altitudes over forest areas," Forest Research Papers, 79(1), 59-68, 2019, doi:10.2478/frp-2018-0007.

[3] S. Zhang, and J. Yao, Single image dehazing using fixed points and nearestneighbor regularization, Computer Vision - ACCV 2016 Workshops Springer, 2016.

[4] K. He, J. Sun, and X. Tang, "Single image haze removal using dark channel prior," in IEEE transactions on pattern analysis and machine intelligence 
33.12: 2341-2353, 2011.doi:10.1109/TPAMI.2010.168.

[5] Y. Xu, J. Wen, L. Fei, Z. Zhang, "Review of Video and Image Defogging Algorithms and Related Studies on Image Restoration and Enhancement," IEEE Access, 4(c), 165-188, 2016, doi:10.1109/ACCESS.2015.2511558.

[6] S. Lee, S. Yun, J.H. Nam, C.S. Won, S.W. Jung, "A review on dark channel prior based image dehazing algorithms," Eurasip Journal on Image and Video Processing, 2016(1), 1-23, 2016, doi:10.1186/s13640-016-0104-y.

[7] Y. Huang, W. Ding, H. Li, "Haze removal for UAV reconnaissance images using layered scattering model," Chinese Journal of Aeronautics, 29(2), 502511, 2016, doi:10.1016/j.cja.2016.01.012.

[8] C. Pat S. Jr., Atmospheric, solar, and M.T.F. corrections for ERTS digital imagery, S.l. : s.n., 1975.

[9] R. Richter, "Atmospheric correction of DAIS hyperspectral image data," Computers and Geosciences, 22(7), 785-793, 1996, doi:10.1016/00983004(96)00016-7.

[10] D. Chowdhury, M. Sarkar, M. Z. Haider, S. A. Fattah, and C. Shahnaz, "Design and implementation of a cyber-vigilance system for anti-terrorist drives based on an unmanned aerial vehicular networking signal jammer for specific territorial security," in IEEE Region 10 Humanitarian Technology Conference (R10-HTC), 2017. doi:10.1109/R10-HTC.2017.8288995.

[11] S.A. Fattah, M.Z. Haider, D. Chowdhury, M. Sarkar, R.I. Chowdhury, M.S. Islam, R. Karim, A. Rahi, C. Shahnaz, "An aerial landmine detection system with dynamic path and explosion mode identification features," in GHTC 2016 - IEEE Global Humanitarian Technology Conference: Technology for the Benefit of Humanity, Conference Proceedings, 745-752, 2016, doi:10.1109/GHTC.2016.7857361.

[12] H. Liu, J. Yang, Z. Wu, Q. Zhang, "Fast single image dehazing based on image fusion," Journal of Electronic Imaging, 24(1), 013020, 2015, doi:10.1117/1.jei.24.1.013020.

[13] E. Woloszyn, An Overview of Meteorology and Climatology, Ph.D. Thesis Gdansk University of Technology, Poland, 2009.

[14] K. He, J. Sun, X. Tang, "Guided image filtering," IEEE Transactions on Pattern Analysis and Machine Intelligence, 35(6), 1397-1409, 2013, doi:10.1109/TPAMI.2012.213.

[15] R. Fattal, "Single image dehazing," ACM Transactions on Graphics, 27(3), 2008, doi:10.1145/1360612.1360671. 